\title{
Spectral Analysis and Design Approach for High Force-to-Volume Extrusion Damper-based Structural Energy Dissipation.
}

\author{
Geoffrey W. Rodgers, John B. Mander, J. Geoffrey Chase, Rajesh P Dhakal, Nicholas C. Leach, \\ Caleb S. Denmead \\ Departments of Mechanical and Civil Engineering, University of Canterbury, Private Bag 4800, Christchurch, \\ New Zealand
}

Email: geoff.chase@canterbury.ac.nz

(Corresponding Author: Associate Professor J. Geoffrey Chase (Details above)) 


\begin{abstract}
High force to volume extrusion damping devices can offer significant energy dissipation directly in structural connections and significantly reduce seismic response. Realistic force levels up to $400 \mathrm{kN}$ have been obtained experimentally validating this overall concept. This paper develops spectral-based design equations for their application. Response spectra analysis for multiple, probabilistically scaled earthquake suites are used to delineate the response reductions due to added extrusion damping. Representative statistics and damping reduction factors are utilised to characterise the modified response in a form suitable for current performance based design methods. Multiple equation regression analysis is used to characterise reduction factors in the constant acceleration, constant velocity, and constant displacement regions of the response spectra.
\end{abstract}

With peak device forces of $10 \%$ of structural weight, peak damping reduction factors in the constant displacement region of the spectra are approximately $6.5 \mathrm{x}, 4.0 \mathrm{x}$, and $2.8 \mathrm{x}$ for the low, medium and high suites respectively. At $\mathrm{T}=1 \mathrm{sec}$, these values are approximately $3.6 \mathrm{x}$, $1.8 \mathrm{x}$, and 1.4x respectively. The maximum systematic bias introduced by the use of empirical equations to approximate damping reduction factors in design analyses is within the range of $+10 \%$ to $-20 \%$. The seismic demand spectrum approach is shown to be conservative across a majority of the spectrum, except for large added damping between $\mathrm{T}=0.8-3.5 \mathrm{sec}$, where it slightly underestimates the demand up to a maximum of approximately $10 \%$. Overall, the analysis shows that these devices have significant potential to reduce seismic response and damage at validated prototype device force levels. 


\subsection{Introduction}

Earthquakes can cause significant structural damage, especially in beam/column connections where movement causes major damage and degradation of structural integrity. The associated damage can be difficult and expensive to repair even though modern structural design methods seek to localise damage to specific areas, such as plastic hinge zones. Although damage also provides significant energy dissipation during the event, it would be desirable to achieve this energy dissipation without permanent structural damage.

In recent years, research in the development of new structural technologies has made significant progress in the reduction of the overall seismic response of civil structures. Active and semi-active systems have been the focus of much of this research. These systems can produce significant reductions in seismic response, but have several key drawbacks compared to a passive system. However, even semi-active systems still involve significant added complexity. A passive system does not require any of these sensing, computing or actuation mechanisms and can thus be relatively inexpensive to manufacture and implement. Moreover, passive control systems may also require little significant regular maintenance. Passive systems already developed for energy absorption and base-isolation applications include lead extrusion dampers [1] and lead-rubber bearings [2, 3].

Lead extrusion dampers used in previous structural applications have been quite large due to the relatively low internal pressures. Their shear size is often considered to be an impediment and thus prevents implementation into several possible applications. Therefore, a compact, high internal pressure damper would enable significant potential new structural applications, particularly in extending applications to emplace dampers within structural connections. Hence, such a damper must be significantly smaller than existing extrusion dampers to broaden possible structure placement into situations with tight volume constraints. However, they must still provide the force capacity and energy dissipation of 
existing, much larger, dampers. More specifically, a high force to volume extrusion damper, relative to existing devices, is required to broaden the application of this technology.

One possible application for extrusion damping is placement into bridge piers, where the load taken by the extrusion damper reduces demand on the columns from transverse loading, as presented schematically in Figure 1a. This approach could be adapted as a supplementary device to retrofit existing seismically vulnerable piers. Alternatively, the dampers could be used in conjunction with bridge piers that use Damage Avoidance Design, as these piers often have low inherent structural damping.

A high force to volume ratio also enables such extrusion dampers to be directly fitted into the columns of steel frame buildings, providing hysteretic energy absorption in the beam-column connections without yielding of the main structural steel elements of the frame. A schematic representation of this application is presented in Figure 1b, where a lead extrusion damper is fitted to an exterior rocking connection.

A further application, enabled by reduced device size, is placement into reinforced concrete beam-column connections, to reduce spalling of the concrete and yielding of the reinforcing bars. This approach is also very amenable to joints that use Damage Avoidance Design (DAD). A schematic representation of this situation is presented in Figure 1c, along with a photograph of the experimental setup of a rocking DAD joint fitted with a lead extrusion damper in Figure 1d. To further illustrate the size of the dampers, Figure 1e presents a photograph of the $120 \mathrm{kN}$ lead extrusion damper of Figure 1d along with some common items to indicate scale. Figure 1e presents a photograph of a $250 \mathrm{kN}$ damper to be embedded in the jointed pre-cast connection of Figure 1f, again shown with common items for scale. The joint of Figure 1f is awaiting a final closure pour to embed the damper into the beam end zone. 
The efficacy and potential of high force to volume lead extrusion devices is shown by the construction and testing of a full scale prototype lead-extrusion damper, to verify force levels and hysteretic behaviour [4]. The velocity dependent elastic-plastic behaviour demonstrated by these lead extrusion dampers is shown to provide an almost ideal square hysteresis loop. As a result, it encloses the maximum possible area within the forcedisplacement plane, providing the maximum energy dissipation achievable per cycle in a consistent and repeatable manner.

The primary goal of this research is to investigate the impact on structural response of high force to volume extrusion devices providing high supplemental damping. It also demonstrates a compact high internal pressure extrusion damper with high force capacity to experimentally validate the concept examined analytically. The analytical investigation is performed using response spectra for a seismically excited, linear elastic, equivalent single degree of freedom structure, similar to that presented schematically in Figure 1a. Seismic response simulations are performed using the three suites of probabilistically scaled earthquake records from the SAC project $[5,6]$. Performance is measured by the use of appropriate log-normal statistics, focusing on spectral acceleration and the corresponding reduction factors. Multiple equation regression analysis is then utilised to develop expressions to estimate the damping reduction factors for structural design purposes. Finally, the systematic bias introduced by incorporating these approximate equations for the damping reduction factors into design analyses is also determined.

\subsection{Device Design and Analysis}

\subsection{Experimental device design.}

Lead extrusion dampers can be categorised into two groups based upon fundamental design differences. These two groups are the constricted tube type, and the bulged shaft type 
[1]. Both types utilise the same basic concept of providing a resistive force by plastically extruding the lead through an orifice created by an annular restriction. For a constricted tube damper, the orifice is created by a constriction on the bore of the outer cylinder. In contrast, bulged shaft dampers utilise a streamlined bulge on the central shaft. The relative merits of each are documented [1] and focus primarily upon ease of manufacture and the ability to achieve predictable and repeatable performance.

The prototype constructed and tested within this research is a bulged shaft lead extrusion damper with an internal diameter of $90 \mathrm{~mm}$, and internal length of $110 \mathrm{~mm}$, a shaft diameter of $30 \mathrm{~mm}$ with a smooth $30 \mathrm{~mm}$ long, $50 \mathrm{~mm}$ diameter bulge. A bulged shaft design is selected for low cost and ease of manufacture. Initial testing is performed as low speed pseudo-static testing, and then over a range of velocities to investigate velocity dependence.

\subsection{Theoretical Device Characterisation for Design Applications}

A lead extrusion damper can be modelled as a non-linear velocity dependent damper, where the damper force, $F$, is defined:

$$
F=C_{\alpha} \dot{x}^{\alpha}
$$

where $C_{\alpha}=$ a constant dependent on device geometry and working material; $\dot{x}=$ the velocity of the damper shaft; and $\alpha=$ the velocity exponent.

Pekcan et al [7] investigated the seismic performance of a class of dampers conforming to Equation (1). Across a broad spectrum of response, they showed that such non-linear dampers can be characterised in terms of equivalent viscous damping, $\xi_{d}$. 


$$
\xi_{d}=\frac{\varepsilon}{1+\alpha}\left(\frac{2 \pi}{0.75}\right)^{0.15(\alpha-1)} g^{0.5(0.85 \alpha+0.15)} S_{d}^{0.5(1.15 \alpha-0.15)} C_{d}^{0.5(0.85 \alpha-1.85)}
$$

where $S_{d}=$ the spectral displacement $(\mathrm{m}), C_{d}=V_{b} / W=$ the normalised base-shear demand (dimensionless), $V_{b}=$ the total base shear force $(\mathrm{N}), W=$ the total seismic weight $(\mathrm{N})$, $g=$ the acceleration due to gravity $\left(\mathrm{m} / \mathrm{s}^{2}\right)$, and $\varepsilon=$ the non-dimensional damper capacity defined by:

$$
\varepsilon=\frac{C_{\alpha} \dot{x}_{r e f}^{\alpha}}{W}
$$

where $\dot{x}_{r e f}=$ a reference velocity used as a standard when testing velocity sensitive dampers. By convention, and for convenience, several manufacturers take this value as $\dot{x}_{r e f}=1 \mathrm{~m} / \mathrm{s}$. It should be noted that Equation (2) above is empirical, but it has been derived based upon a broad spectrum of earthquake records and uses a power absorption equivalence instead of an energy equivalence.

For bulged shaft lead extrusion dampers a value of $\alpha$ in the range of $\alpha=0.11$ to 0.15 is appropriate based upon previous research [1] and experimental data. Thus, for convenience, by substituting $\alpha=0.13$, and $g=9.81 \mathrm{~m} / \mathrm{s}^{2}$ into Equation (2), damping can be found independent of the spectral displacement amplitude, $S_{d}$ :

$$
\xi_{d_{\alpha=0.13}}=\frac{0.90}{C_{d}^{0.87}} \varepsilon
$$


where $\varepsilon$ can be thought of as the normalised damper capacity, defined as the peak damper force for a full speed test. Typically, $\varepsilon$ is defined with respect to the structural weight as a convenient reference, as defined in Equation (3). This result shows that the equivalent viscous damping for a bulged shaft type of lead extrusion damper is invariant to the displacement magnitude, as might be expected.

\subsection{Experimental Device and Model Verification}

Low velocity experiments were performed on a full scale prototype to investigate the hysteretic behaviour, and ensure realistic force levels could be obtained reliably and repeatedly. The hysteretic behaviour was found to closely follow the velocity dependent elastic-plastic behaviour presented in other research [1] and modelled for theoretical simulations. The force levels for the prototype were on the order of $250-300 \mathrm{kN}$, representing a realistic force level for the structural applications presented in Figures 1a-c. A typical force-displacement plot for the prototype device is presented in Figure 2 [4], and encloses nearly the largest possible area. Consequently, it dissipates the almost optimal amount of hysteretic energy possible, between the force and displacement shown.

This result demonstrates the advantages of incorporating extrusion damping into key structural design areas to obtain the corresponding large energy dissipation. Experimental testing of the full-scale prototype has been performed at several different velocities, as presented in Figure 3. Also presented in Figure 3 is a velocity dependent device model conforming to Equation (1), where the value of $\alpha$ is set to 0.12 as presented in Cousins et al. [1] and used in the following spectral investigation. The value of the device constant, $C_{\alpha}$ was then calculated based on a least squares best fit. This figure indicates that the velocity dependence of the prototype device can be well modelled by this equation. It should be noted that the maximum test velocity in Figure 3 is $1.3 \mathrm{~mm} / \mathrm{s}$, considerably lower than the reference 
velocity of $1 \mathrm{~m} / \mathrm{s}$ used in Equation (3). Higher speed tests have been undertaken up to velocities of $16-20 \mathrm{~mm} / \mathrm{s}$ with no change in device characteristics. Limitations in testing equipment has prevented the authors undertaking higher speed tests. This area is the subject of ongoing research. Interested readers are also directed to the work of Robinson and Greenback [8] and Cousins and Porritt [1], where testing of similar devices is undertaken at velocities up to approximately $1 \mathrm{~m} / \mathrm{s}$.

\subsection{Spectral Analysis of Seismic Response Reduction for Design}

\subsection{Structure and modelling}

The structure modelled for spectral analysis and statistical quantization of the effectiveness of extrusion damping is based on an equivalent single-degree of freedom (SDOF) system similar to that presented in Figure 1a. Internal, structural viscous damping equal to $5 \%$ of critical is assumed. Additional damping in this analytical investigation is provided by a non-linear bulge-shaft type lead extrusion damper, with a velocity exponent of $\alpha=0.12$ [1]. The non-dimensional damper capacity, or force ratio, $\varepsilon$, from Equation (3), for the extrusion dampers is parametrically set at $1,2,5$ and $10 \%$ of total structural weight. The value of the device constant $C_{\alpha}$ in Equation (1) is calibrated to give the prescribed force level, corresponding to a percentage of structure weight, at a reference velocity of $1 \mathrm{~m} / \mathrm{s}$.

The structure model is utilised to create response spectra for all earthquake records in the three suites from the SAC project $[5,6]$. Each suite includes ten different time histories, with two orthogonal directions for each history. The low, medium and high suites represent ground motions having probabilities of exceedance of $50 \%$ in 50 years, $10 \%$ in 50 years, and $2 \%$ in 50 years respectively. These motions are generally intended to be representative of design objectives of Immediate Occupancy (IO), Life Safety (LS), and Collapse Prevention (CP), respectively. The acceleration histories have been scaled to approximately conform to 
the 1997 NEHRP (that is similar in shape to the Newmark and Hall spectra) design spectrum for firm soil at the specified return periods. Thus variations between records within a suite are indicative of record-to-record randomness at a given site. The time histories for both the low and medium suite are all derived from actual recordings of crustal earthquakes on stiff soils. For the high suite, five of the time histories are from recorded near-fault events, and the other five are synthetically generated. The SAC scaling procedure involved constructing a target response spectrum for each of the three excitation levels, by converting data from USGS probabilistic seismic hazard maps at the site to a spectra representing stiff local soil conditions. Individual time histories were scaled by a single scaling factor that minimised the root-mean-square error between the target spectrum and the average response spectrum of the two horizontal components of the time history assuming lognormal distribution of amplitudes.

\subsection{Statistical Analysis of Results}

Representative statistics were evaluated from the individual structural responses for the 20 earthquake records within each suite. Statistical analysis showed the response quantities conform to a log-normal distribution. The two parameters that characterise the lognormal distribution are the median, $\hat{x}$, and log-normal coefficient of variation (dispersion factor), $\beta$, were thus determined for each suite and overall.

$$
\hat{x}=\exp \left(\frac{1}{n} \sum_{i=1}^{n} \ln \left(x_{i}\right)\right)
$$

where $x_{i}$ are the response metric for ground motion $i$, and $n=20$ for a given suite. 


$$
\beta=\sqrt{\frac{1}{n-1} \sum_{i=1}^{n}\left(\ln \left(x_{i} / \hat{x}\right)\right)^{2}}
$$

To illustrate the change in response variables across the entire response spectrum both the median and dispersion factors are plotted against structural natural period.

\subsection{Analysis Methodology}

The acceleration spectral response is measured and gives an indication of the performance of the lead extrusion damper at preventing damage to the non-structural components of the building. The geometric mean, or median, response of the earthquake records within each suite is plotted for non-dimensional damper capacities of $\varepsilon=0,1,2,5$, $10 \%$, to indicate the reductions achieved with the addition of the extrusion dampers.

The coefficient of variation, or dispersion factor is presented to give an indication of the spread of the results within each suite. Reduction factors from the $\varepsilon=0$ case are calculated for the geometric mean spectral acceleration response within each suite to more clearly represent the response reductions achieved with different levels of added nondimensional damper capacity, $\varepsilon$. The geometric mean response for the low and high suite is then normalised to the geometric mean from the medium suite at each value of nondimensional damper capacity, $\varepsilon$. This last analysis indicates the relative performance of the extrusion dampers when subjected to far field and near field ground motions.

To relate the results of the spectral analysis to design guidelines, regression analysis is used to develop equations to approximate the damping reduction factors. Multiplicative error factors are then calculated to indicate the error introduced by using these equations to approximate the reduction factors calculated from the spectral analyses. Finally, the seismic demand spectrum is calculated for all levels of damper capacity, $\varepsilon$, and the error factors 
between these spectra and the overall geometric mean spectra from the medium suite are calculated. These error factors indicate whether the seismic demand spectrum design approach is conservative.

\subsection{Spectral Analysis Results}

\subsection{Spectral Response Simulation Results}

The individual spectral response for each of the earthquakes in all 3 suites was simulated with the addition of lead extrusion dampers utilising characteristic force levels, $\varepsilon=$ $0,1,2,5,10 \%$ of total structural weight. Note that the $\varepsilon=0$ case is done to show the response without any added devices.

The geometric mean, or median, response spectra for each suite is presented in the left column of Figure 4 and clearly shows the response reductions achieved by the addition of extrusion damping. The peak response values decrease notably across all suites as the nondimensional damper capacity, $\varepsilon$, increases from $0 \%$ to a maximum of $10 \%$ of total structural weight. The median response spectrum for each suite is presented for $\varepsilon=0,1,2,5$, and $10 \%$ of structural weight. To indicate the effect that extrusion damping has on the spread of the results, the log-normal coefficient of variation, or dispersive factor $\beta$, is plotted against structural natural period for the same cases, and presented in the right column of Figure 4.

The reductions achieved across a suite of earthquakes by the addition of extrusion damping can also be represented by the use of damping reduction factors. These factors are a clear way of presenting the reductions that can be achieved across a response spectrum. The damping reduction factors for the geometric mean responses are presented in Figure 5, where the reduction factor is a divisive factor, and represents the factor by which division of the uncontrolled response yields the response with the added damper. 
The damping reduction factors are characterised into three regions based upon existing bifurcation points. These three regions are the constant displacement, constant velocity, and constant acceleration regions, and are defined graphically in Figures 6. The largest damping reduction factors in Figure 5 are seen in the constant displacement region of the spectra for the low suite, with an average reduction factor of approximately 6.4 for a $10 \%$ structural weight extrusion damper. This value compares with average reduction factors for the same force capacity of approximately 3.75 and 2.5 for the same region of the medium and high suite spectra, respectively. These reduction factors represent substantial decreases in the spectral acceleration response with the use of realistically sized extrusion dampers.

The reduction factors in the range of $T \approx 1 \mathrm{sec}$, a period typical of a family of medium rise structures, are approximately 3.6, 1.8, and 1.4 for the low, medium, and high suite respectively, for a $10 \%$ structural weight damper, as seen in Figures 5a-c. These reduction factors are smaller than those seen in the constant displacement region, but are at a more common period for widespread structural design.

The reduction factors increase approximately linearly with an increasing damper capacity. The average reduction factors for the medium suite in the constant displacement region of the spectrum are approximately $1.2,1.4,2.15$, and 3.8 for non-dimensional damper capacities, $\varepsilon$, equal to $1,2,5$, and $10 \%$ of total structural weight respectively. The overall average reduction factors at a structural period of $T=1 \mathrm{sec}$ are approximately $1.08,1.16$, 1.39 , and 1.80 for the same range of damper capacities, $\varepsilon$. Both regions show a strong linear relationship between the reduction factors and the added damper capacity.

To investigate the suite dependence of the reduction factors, the normalised reduction factors are calculated. These normalised factors are defined as the geometric mean reduction factor derived from the low and high suites and each force level, $\varepsilon$, divided by the geometric mean reduction factors from the medium suite at that force level $\varepsilon$ and across all natural 
periods. This normalisation gives an indication of the relative performance of the extrusion dampers across the different suites, where the results are presented in relation to the medium suite.

The results from this normalisation analysis are presented in Figure 7 and indicate that the dampers produce the biggest reductions in spectral acceleration for the more vibratory ground motions of the low suite. The reduction factors for the low suite are greater than the average reduction factors from the medium suite across the entire spectrum. Normalised reductions for large added damping $(\varepsilon=10 \%)$ for the high suite were as low as 0.6 across a range of the spectrum, indicating that the extrusion dampers are not as well suited to the large pulses of the high suite as they are to more vibratory ground motions of the low suite.

\subsection{Empirical Modelling of Results for Design}

To enable the future applications of lead extrusion dampers to be incorporated into structural design procedures, it is desirable to develop equations that estimate the damping reduction factors. Previous research has utilised non-linear regression analysis to develop a single equation model that approximates the reduction factors across the entire spectrum [9]. This research utilises a new method of segregating the spectrum into three regions based upon the existing bifurcation points, and developing equations for each of these regions.

Although the normalised reduction factor graphs in Figure 7a-d show that there are reasonable variations across the three suites, especially for high additional damping, seismic design procedures are normally based upon earthquake design spectra that have characteristics similar to the medium suite. Thus, equations are fit based to the median reduction factors from the medium suite. The three regions defined are the constant acceleration, constant velocity, and constant displacement portions of the spectra, with the 
bifurcation points occurring at natural periods of 0.4 and 3.0 seconds, as shown in Figure 6 . Linear regression analysis is employed to develop equations for the constant acceleration and constant displacement regions, and linear interpolation between the values at $T=0.4 \mathrm{sec}$ and $T=3.0 \mathrm{sec}$ is utilised to define the constant velocity region. Regression analysis gives the equation for the constant acceleration region of the medium suite geometric mean reduction factor spectra as:

$$
B_{a}=1+4.43 \varepsilon \quad(T<0.4 \mathrm{sec})
$$

where $\varepsilon$ is the damper reference force as a fraction of total structural weight.

Similarly, the equation for the constant displacement region of the reduction factors for the medium suite geometric mean response is:

$$
B_{d}=1+26.7 \varepsilon \quad(T \geq 3 \mathrm{sec})
$$

Linear interpolation between the values at 0.4 second and 3.0 seconds natural period, given by Equations (7) and (8) respectively, yields the equation for the constant velocity region:

$$
B_{v}=1+(8.56 T+1.0) \varepsilon \quad(0.4 \leq T<3 \mathrm{sec})
$$

where $T$ is the structural natural period, and $\varepsilon$ is, as before, the damper reference force as a fraction of total structural weight. For clarity, the reduction factors for the medium suite geometric mean response from Figure $5 \mathrm{~b}$ are reproduced with the corresponding plot of the multiple equation model in Figures $8 \mathrm{a}$ and $\mathrm{b}$ respectively. It is worth noting for comparison, that for a structure with a natural period of $1 \mathrm{~s}$, non-dimensional damping capacities of $\varepsilon=1$, 
2, 5 and $10 \%$ of structural weight correspond to total equivalent viscous damping of 7, 9.2, 16.8 , and $33.2 \%$, where these values include the $5 \%$ inherent structural damping assumed in the analysis.

The medium suite geometric mean design spectra for $1,2,5$, and $10 \%$ added extrusion damping are reconstructed from the exact spectra with 5\% internal damping to verify the multiple equation model of the damping reduction factors. To investigate whether the equations derived for the damping reduction factors are suitably accurate for design purposes, it is important to look at the systematic bias that is introduced.

To represent the systematic bias introduced by the use of equations to approximate the damping reduction factors, the multiplicative error factors are calculated, by dividing the reconstructed spectra by the corresponding exact spectra. This gives an indication of the relative error that is introduced by this design approach. The multiplicative error factors are plotted in Figure 9. Results from this analysis indicate that the maximum systematic bias introduced is within the range of $+10 \%$ and $-20 \%$.

\subsection{Design Implementation}

The seismic demand spectrum is useful where a realistic evaluation of the earthquake hazard can be analysed based on the statistical analysis of past earthquake records. The seismic demand spectrum is given by:

$$
C_{d}=\min \left(\frac{F_{a} S_{s}}{B_{a}}, \frac{F_{v} S_{1}}{T B_{v}}, \frac{F_{v} S_{1} T_{d}}{T^{2} B_{d}}\right)
$$

where $F_{a}$ and $F_{v}$ are adjustments on spectral acceleration for short and long periods at different soil classes; $S_{S}$ and $S_{1}$ are spectral acceleration at short periods and the one-second period; $B_{a}, B_{v}$, and $B_{d}$ are the approximate damping reduction factors defined in Equations 
(7)-(9); $T=$ the period of the structure; and $T_{d}=$ the period at the junction of the constant spectral velocity and displacement portions of the spectra. Using Equation (10) accelerationperiod seismic demand spectra for normal soil (where PGA $=F_{v} S_{1}$ and $F_{a} S_{s}=2.5 F_{v} S_{1}$ ) are presented in Figure 10.

To investigate the systematic bias introduced by the use of the seismic demand spectrum, the divisive error factors are calculated. These factors represent the 'exact' median spectra from the medium suite divided by the seismic demand spectra. The divisive error factors are presented in Figure 11. These factors indicate that across most of the spectrum the seismic demand spectrum is conservative as the divisive factors are less than unity, except for large added damping in the range of approximately $0.8-3.5$ second period, where the demand is slightly underestimated, up to a maximum of approximately $10 \%$. Overall, this result indicates that the design approach is generally conservative and reasonably accurate for design purposes.

\subsection{Conclusions}

The high force to volume damper concepts presented provide force capacity, and hence energy dissipation capacity, on the same order of magnitude of significantly larger devices. Their small size enables placement into structural connections and other areas heretofore unavailable, enabling new potential applications at full scale. Comprehensive simulation of structural response across a range of earthquake suites has shown that significant reductions in acceleration response can be achieved with realistically sized dampers. Based on the experimental and analytical investigation described, the following specific conclusions can be drawn: 
- The development of lead extrusion damper technology can produce more compact variants of existing dampers that allow placement into key structural areas that were previously unviable.

- Analysis of response spectra shows that the addition of extrusion damping with force levels up to $10 \%$ of structural weight can have significant impact on the seismic response, with response reduction factors of up to approximately 6.5. This result represents a significant reduction in the spectral acceleration response with realistic damper sizes, as proved by the construction and testing of a full scale prototype.

- Average reduction factors in the constant displacement region of the spectra, with the addition of extrusion damping equal to $10 \%$ of building weight, were approximately 6.4, 3.75, and 2.5 for the low, medium and high suites respectively.

- Reduction factors in the range of $T=1 \mathrm{sec}$, are 3.6, 1.8, and 1.4 for the low, medium, and high suite respectively, for a $10 \%$ structural weight damper. These reduction factors are smaller than those in the constant displacement region, but are at a more common period for widespread structural design.

- Normalised reduction factors show that the dampers perform significantly better across the whole spectrum for the more vibratory ground motions of the low suite than for the large pulses of the high suite.

- Multiple equation regression analysis enables development of empirical expressions to model the effect of response reduction through the lead dampers. Multiplicative error factors indicate that the maximum systematic bias introduced by using the reduction factor equations in design analyses is within the range of $+10 \%$ and $-20 \%$.

- The divisive error factors, representing the exact spectra divided by the seismic demand spectra show that this design approach is conservative and overestimates the demand across the spectrum, except for large added damping between 0.8 and 3.5 
second periods, where it slightly underestimates the demand up to a maximum of approximately $10 \%$.

This research demonstrates that high force to volume extrusion dampers are possible, and can be incorporated into previously unviable areas of structural design, by the development of an extrusion damper with significantly smaller dimensions than those presented in previous work. The results indicate that such devices can significantly impact seismic response and be readily incorporated into accepted design metrics. 


\section{References}

[1] W. J. Cousins and T. E. Porritt, "Improvements to lead-extrusion damper technology," Bulletin of the New Zealand National Society for Earthquake Engineering, vol. 26, pp. 342-348, 1993.

[2] W. H. Robinson, "Recent research and applications of seismic isolation in New Zealand," Bulletin of the New Zealand National Society for Earthquake Engineering, vol. 28, pp. 253-264, 1995.

[3] W. H. Robinson, "Lead-Rubber Hysteretic Bearings Suitable For Protecting Structures During Earthquakes," Earthquake Engineering and Structural Dynamics, vol. 10, pp. 593-604, 1982.

[4] G. W. Rodgers, N. C. Leach, C. S. Denmead, J. G. Chase, and J. B. Mander, "Experimental development and analysis of a high force/volume extrusion damper," Proceedings New Zealand Society for Earthquake Engineering Annual Conference, Napier, New Zealand, March 10-12, 2006.

[5] SAC, "The SAC Steel Project: Seismic Design Criteria for Steel Moment Frame Construction," 1999.

[6] P. Sommerville, N. Smith, S. Punyamurthula, and J. Sun, "Development of Ground Motion Time Histories For Phase II Of The FEMA/SAC Steel Project, SAC Background Document Report SAC/BD-97/04," 1997.

[7] G. Pekcan, J. B. Mander, and S. S. Chen, "Fundamental Considerations for The Design of Non-linear Viscous Dampers," Earthquake Engineering and Structural Dynamics, vol. 4, pp. 251-259, 1976.

[8] W. H. Robinson and L. R. Greenback, "An Extrusion Energy Absorber Suitable for the Protection of Structures During an Earthquake," Earthquake Engineering and Structural Dynamics, vol. 10, pp. 593-604, 1982.

[9] Y.-Y. Lin and K.-C. Chang, "Effects of Site Classes on Damping Reduction Factors," Journal of Structural Engineering, pp. 1667-1675, 2004.

[10] Rodgers, G. W., Chase, J. G., Solberg, K., Mander, J. B., and Dhakal, R. "Analytical modelling of jointed precast concrete beam-to-column connections with different damping systems." New Zealand Society for Earthquake Engineering Annual Conference (NZSEE 07), Palmerston North, New Zealand, 8-pages.

[11] Solberg, K., Bradley, B., Rodgers, G., Mander, J., Dhakal, R., and Chase, J. "MultiLevel Seismic Performance Assessment of a Damage-protected Beam-column Joint with Internal Lead Dampers." New Zealand Society for Earthquake Engineering Annual Conference (NZSEE 07), Palmerston North, New Zealand, 8-pages.

[12] Solberg, K. (2007). "Experimental and Financial Investigations into the further development of Damage Avoidance Design," Master of Engineering (ME) Thesis, University of Canterbury, Christchurch, New Zealand. 
a)

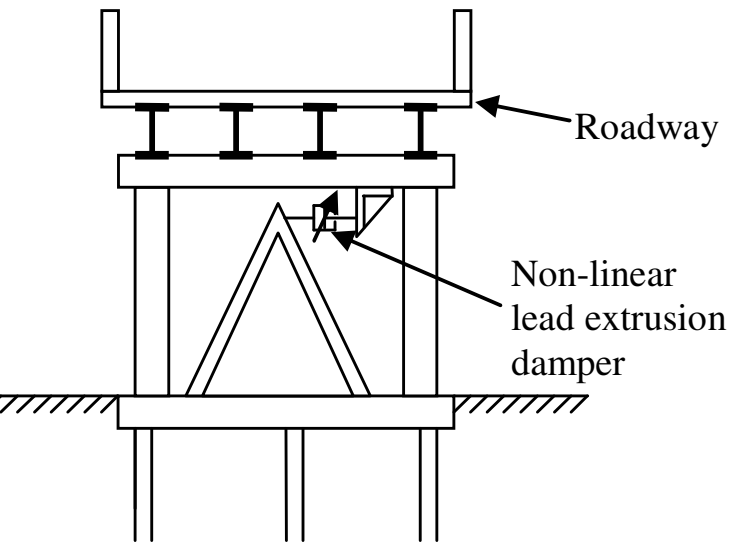

c)

e)

g)
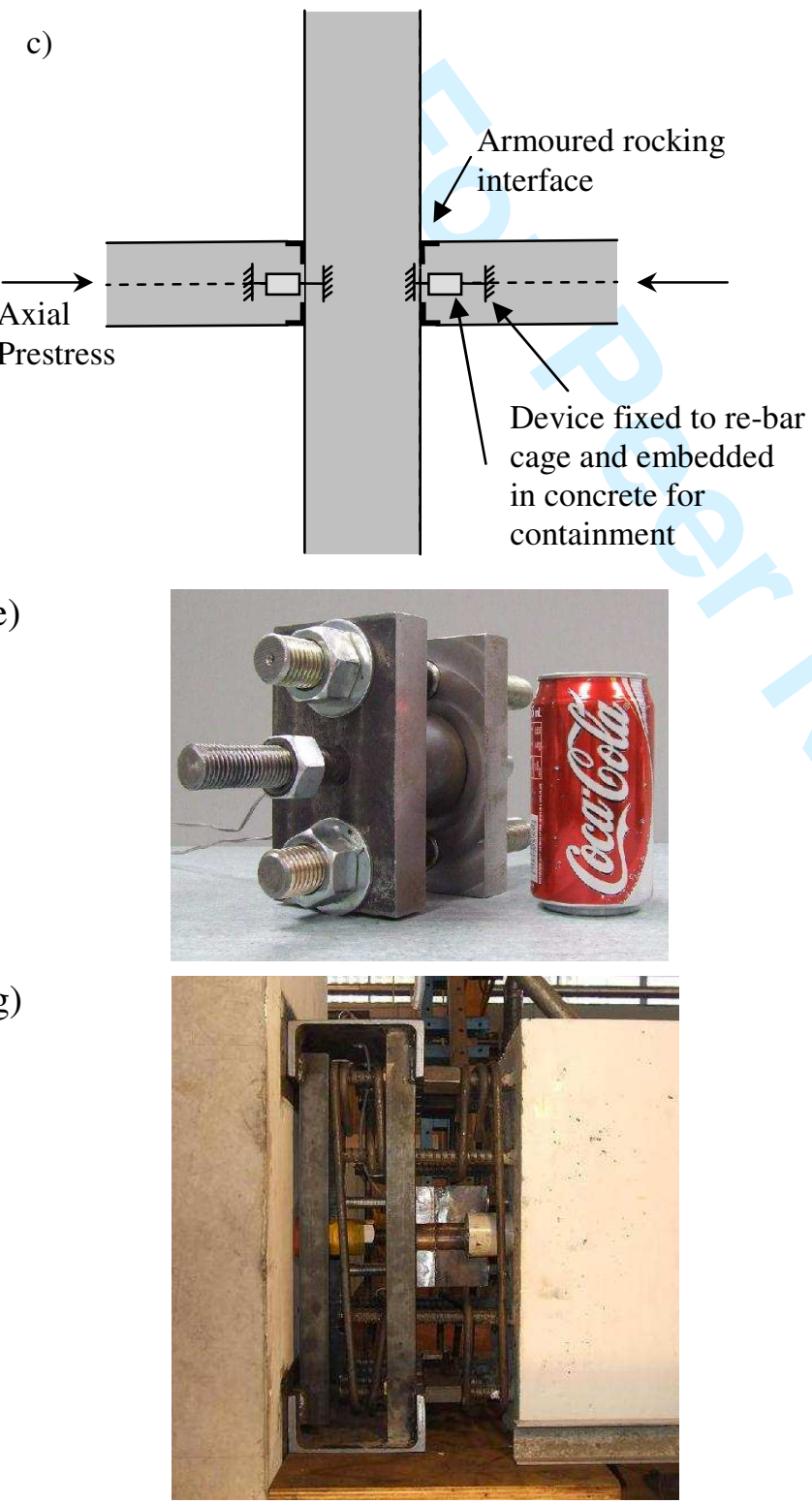

Figure 1: Schematic representation of extrusion damper placements into a) a bridge pier; b) Exterior steel beam column connection with shear connection, c) Reinforced concrete beamcolumn connection, d) photograph of experimental beam-column connection using a lead extrusion damper [10], e) photograph of $120 \mathrm{kN}$ extrusion damper of Figure 1d, f) photograph of $250 \mathrm{kN}$ damper embedded into connection of Figure 1g [11]. g) jointed precast concrete joint with $250 \mathrm{kN}$ damper of Figure $1 \mathrm{f}$ inside joint awaiting closure pour [12]. 


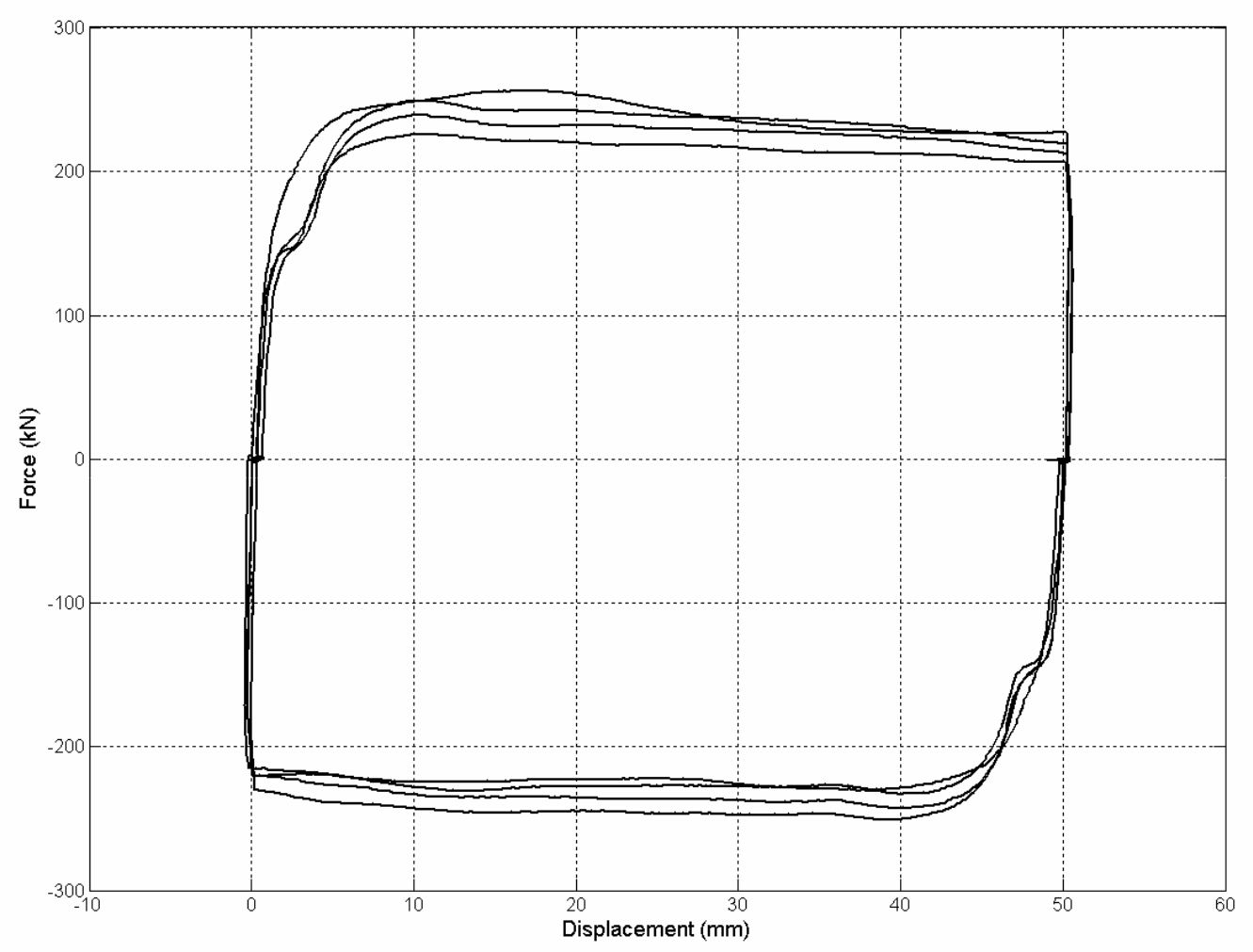

Figure 2: Hysteresis Loop for full scale prototype lead extrusion damper. 


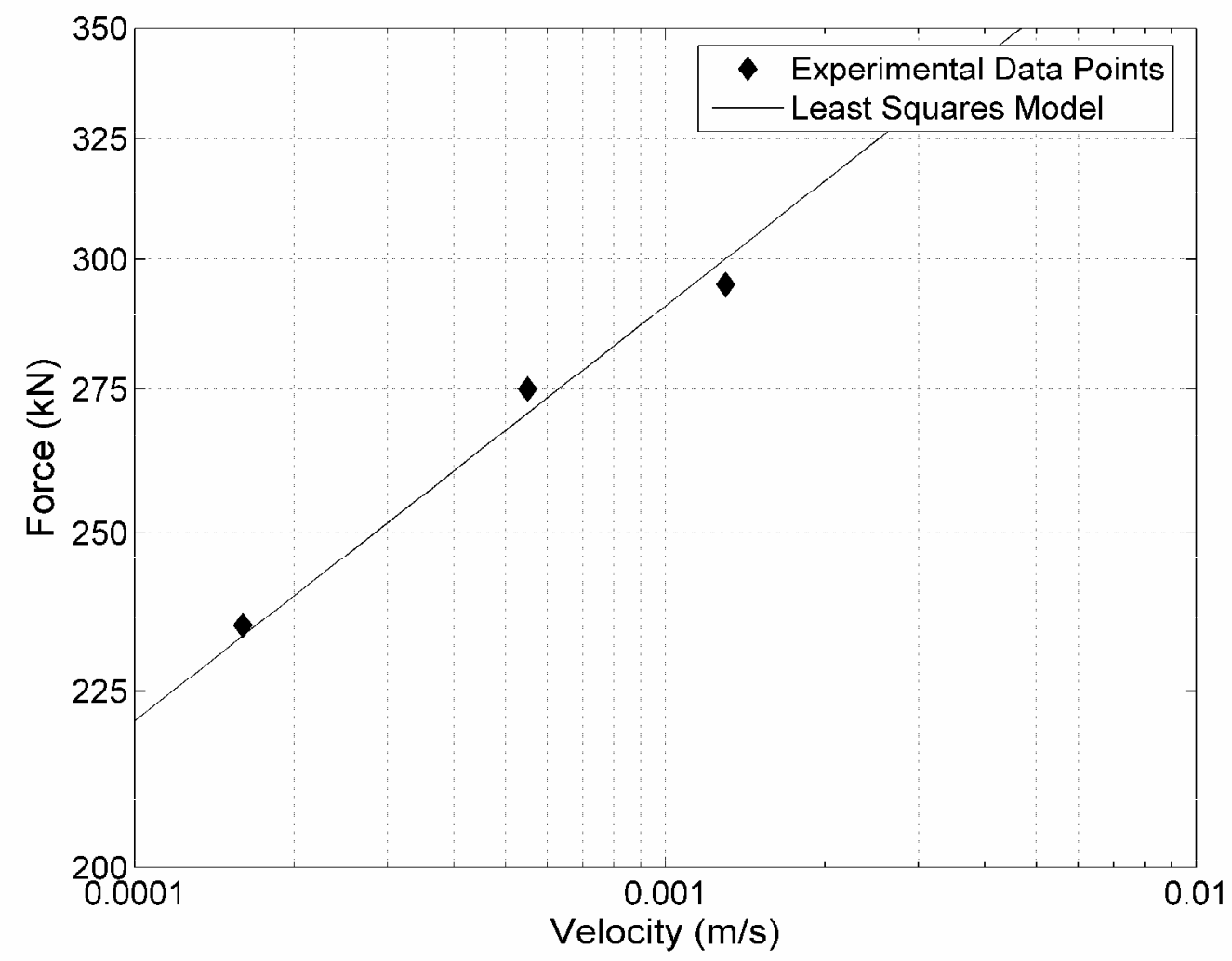

Figure 3: Experimental results for a full-scale prototype tested at a range of velocities, and velocity dependent model from Equation (1), with $\alpha$ set to 0.12 , and $C_{\alpha}$ fitted in a leastsquares sense 

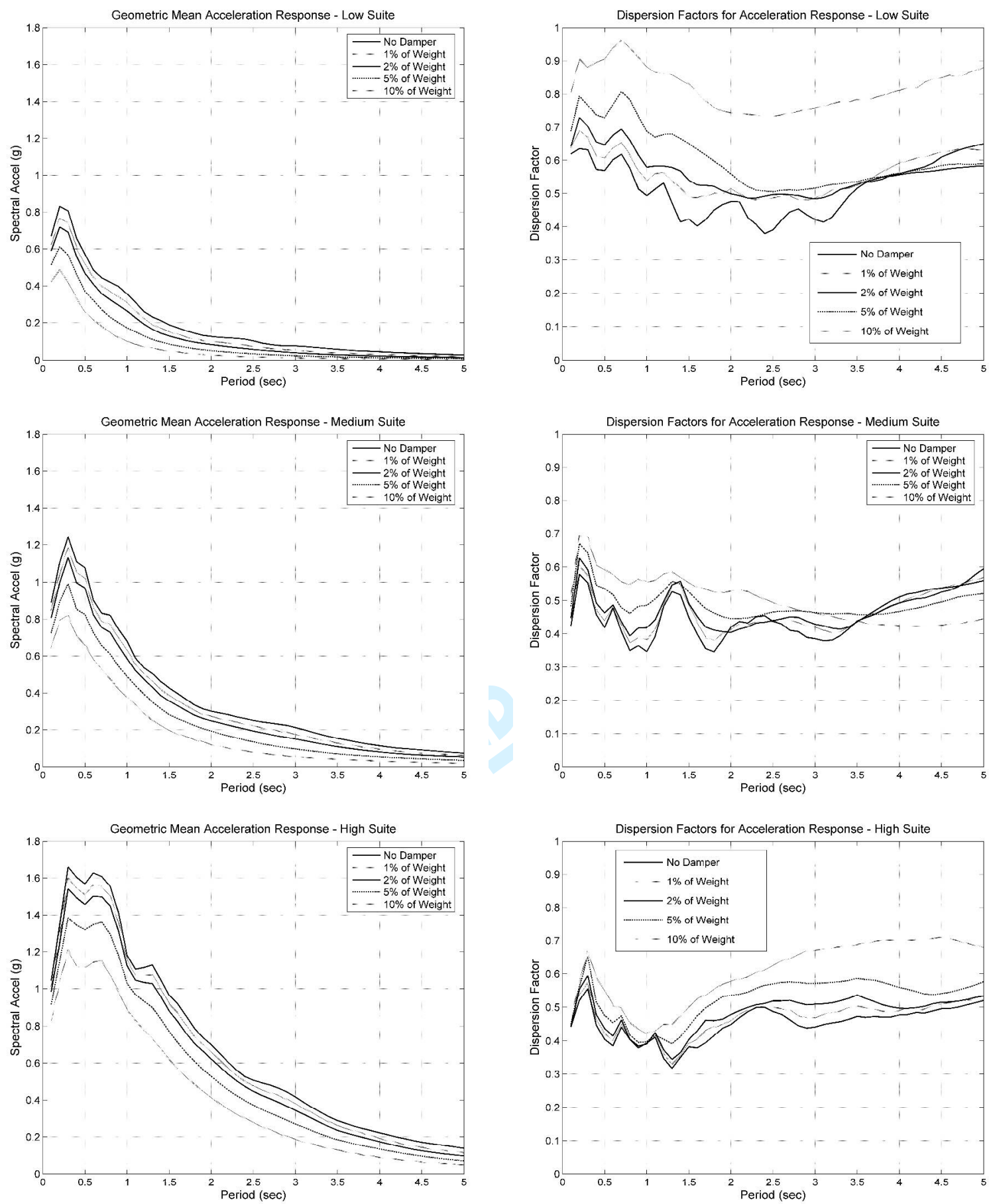

Figure 4: Geometric mean acceleration response spectra and log-normal co-efficient of variation, or dispersion factors, $\beta$, for each suite 
a)

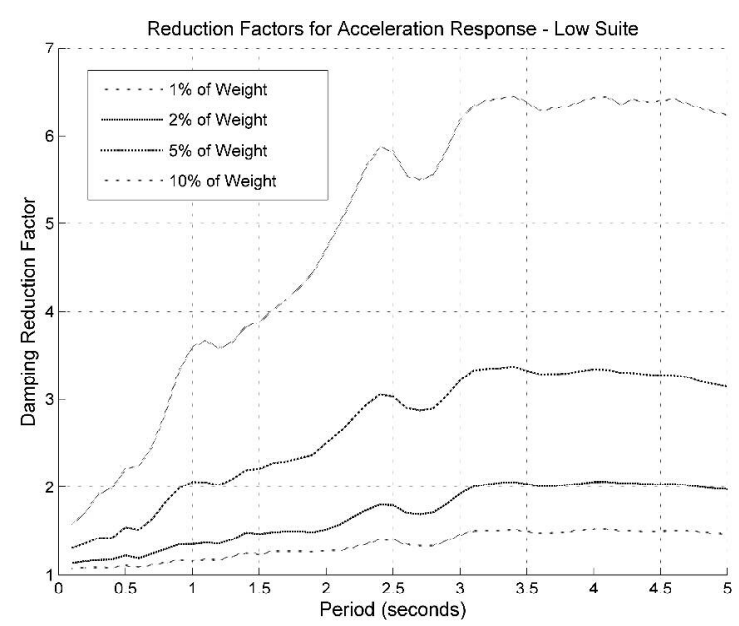

c)

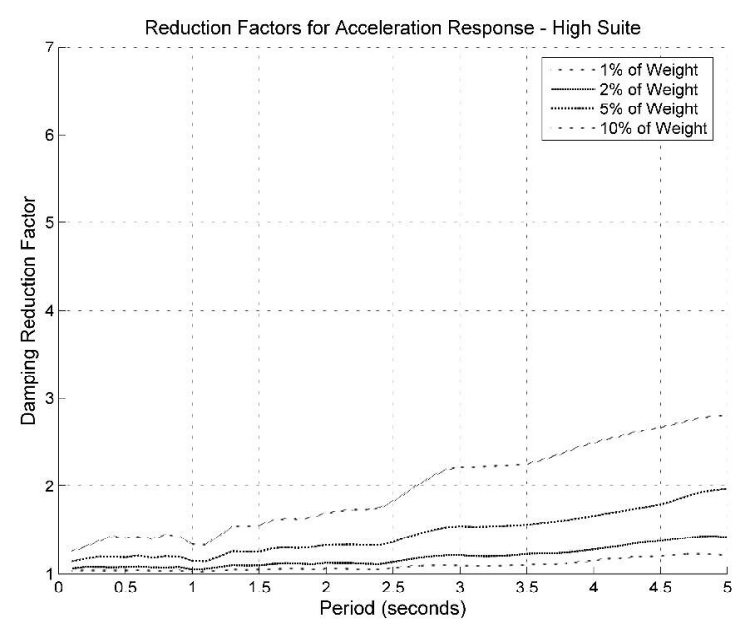

b)

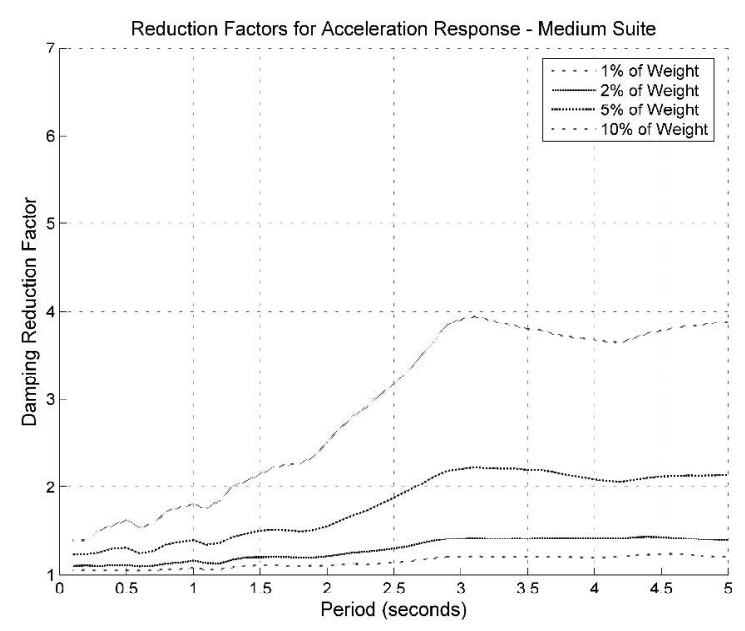

Figure 5: Damping reduction factors for the geometric mean responses for a) the low suite, b) the medium suite, and c) the high suite. 


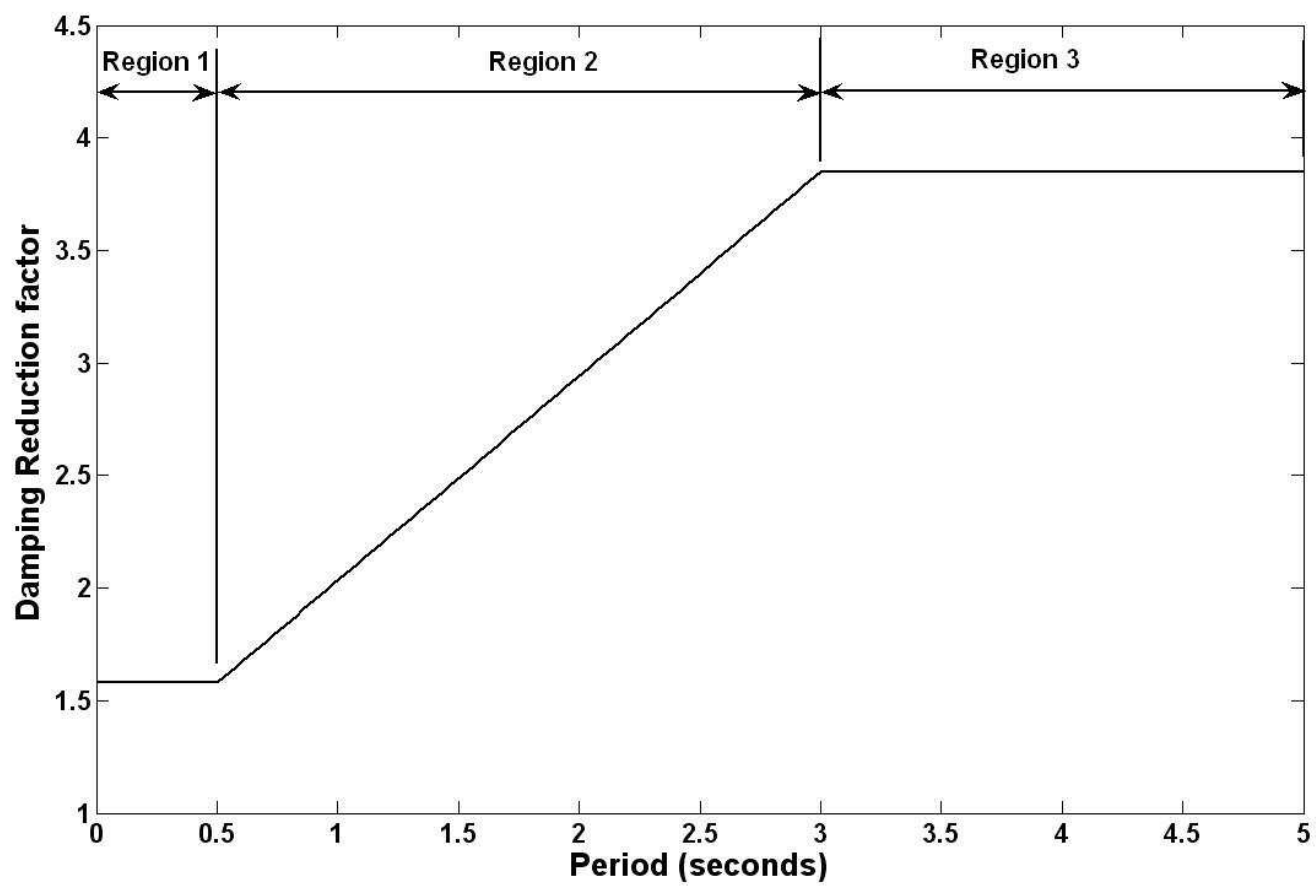

Figure 6: Typical reduction factor plot showing the constant acceleration (Region 1), constant velocity (Region 2), and constant displacement region (Region 3) 
a)

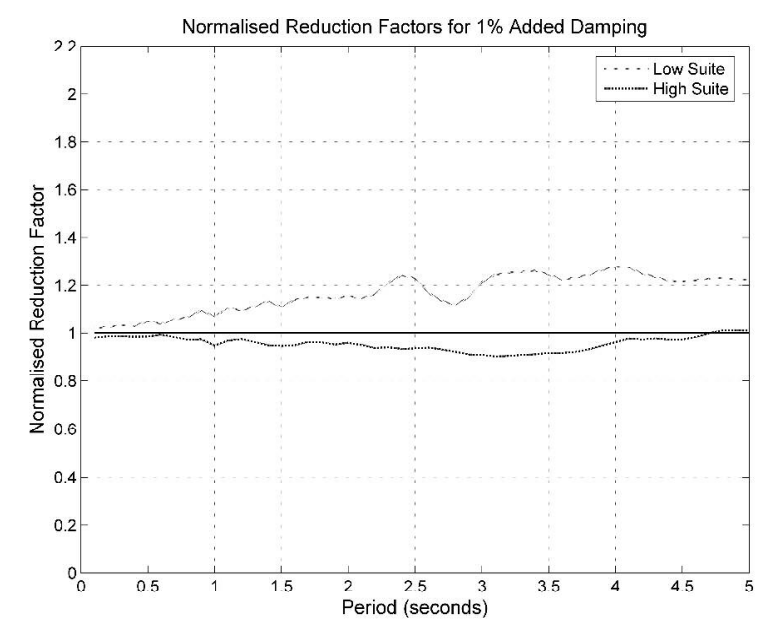

c)

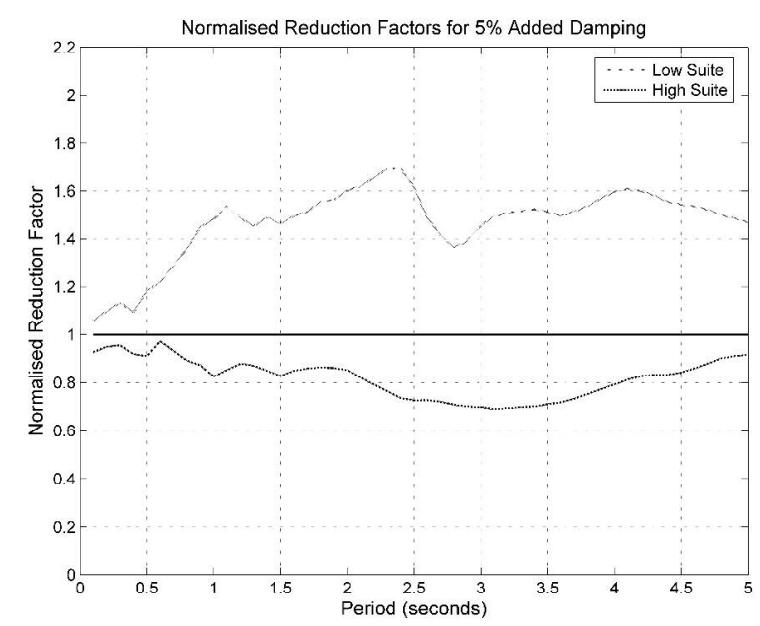

b)

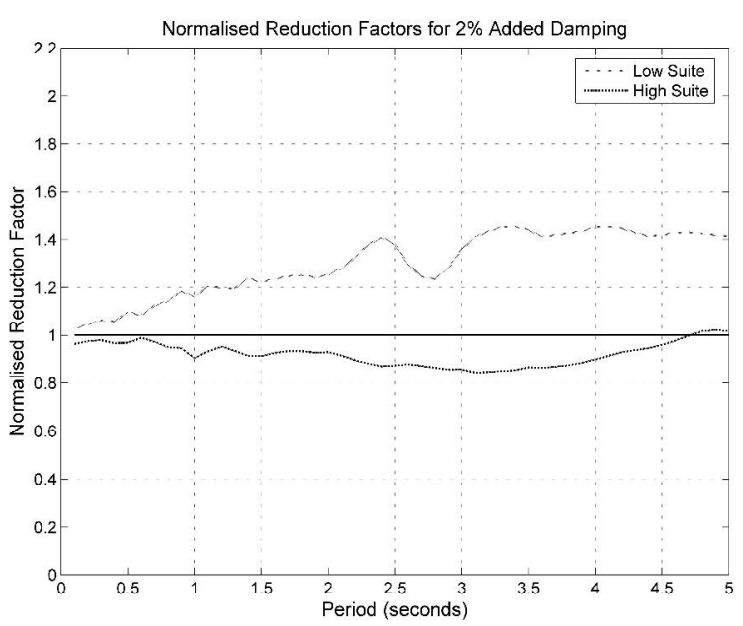

d)

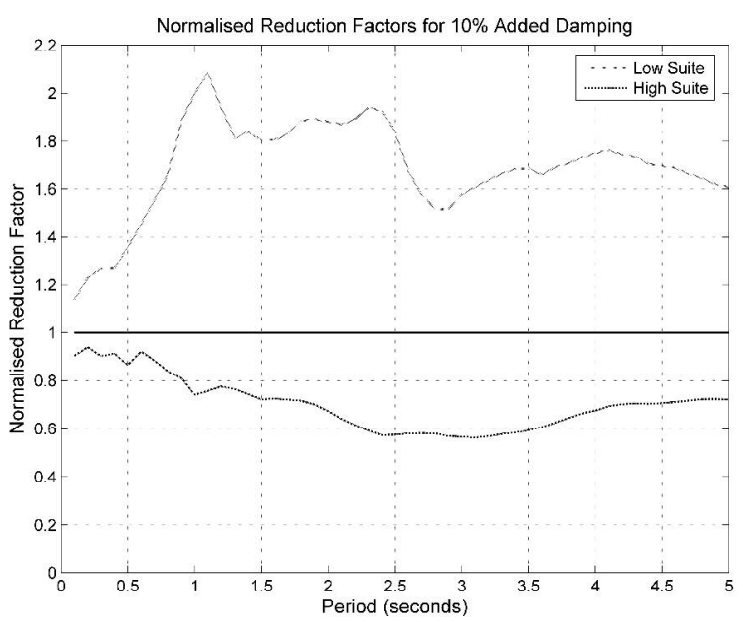

Figure 7: Geometric mean acceleration reduction factors derived from the low and high suites normalised by the geometric mean acceleration reduction factors from the medium suite. 
a)

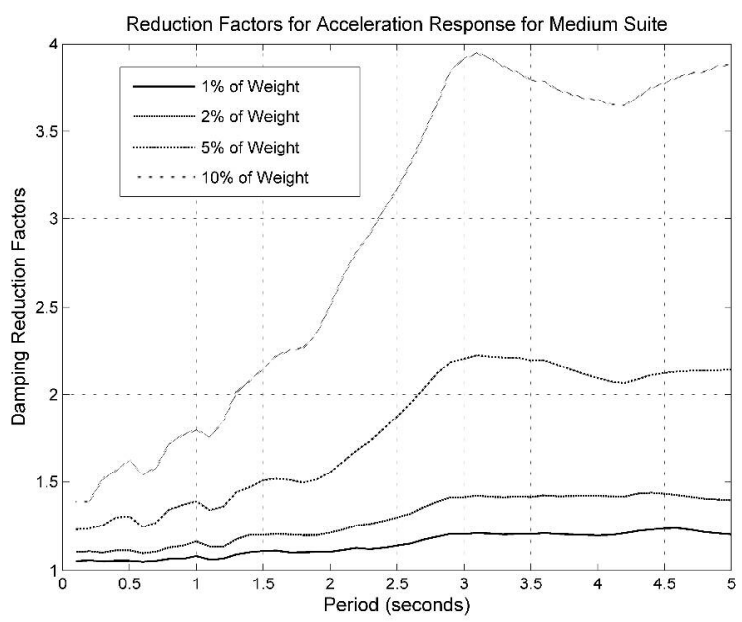

b)

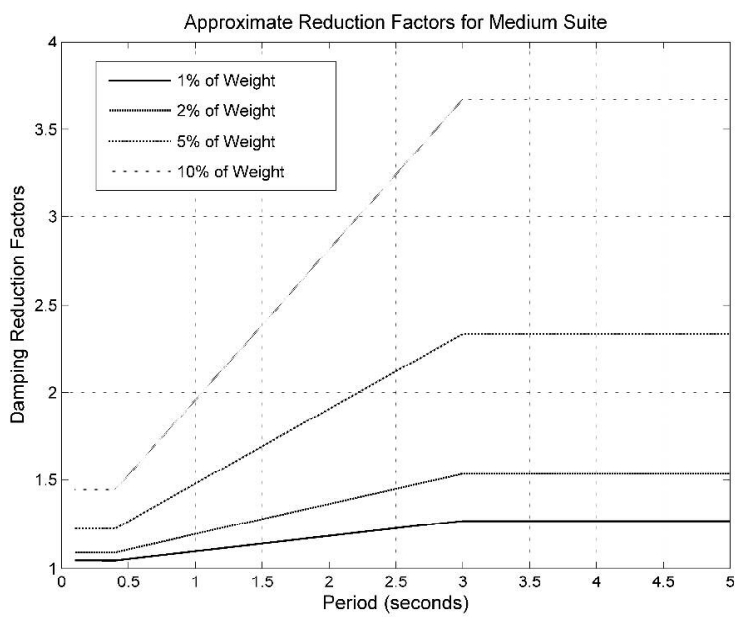

Figure 8: a) Reduction factors for the geometric mean response from the medium suite, and b) Approximate reduction factors defined by the multiple equation model. 


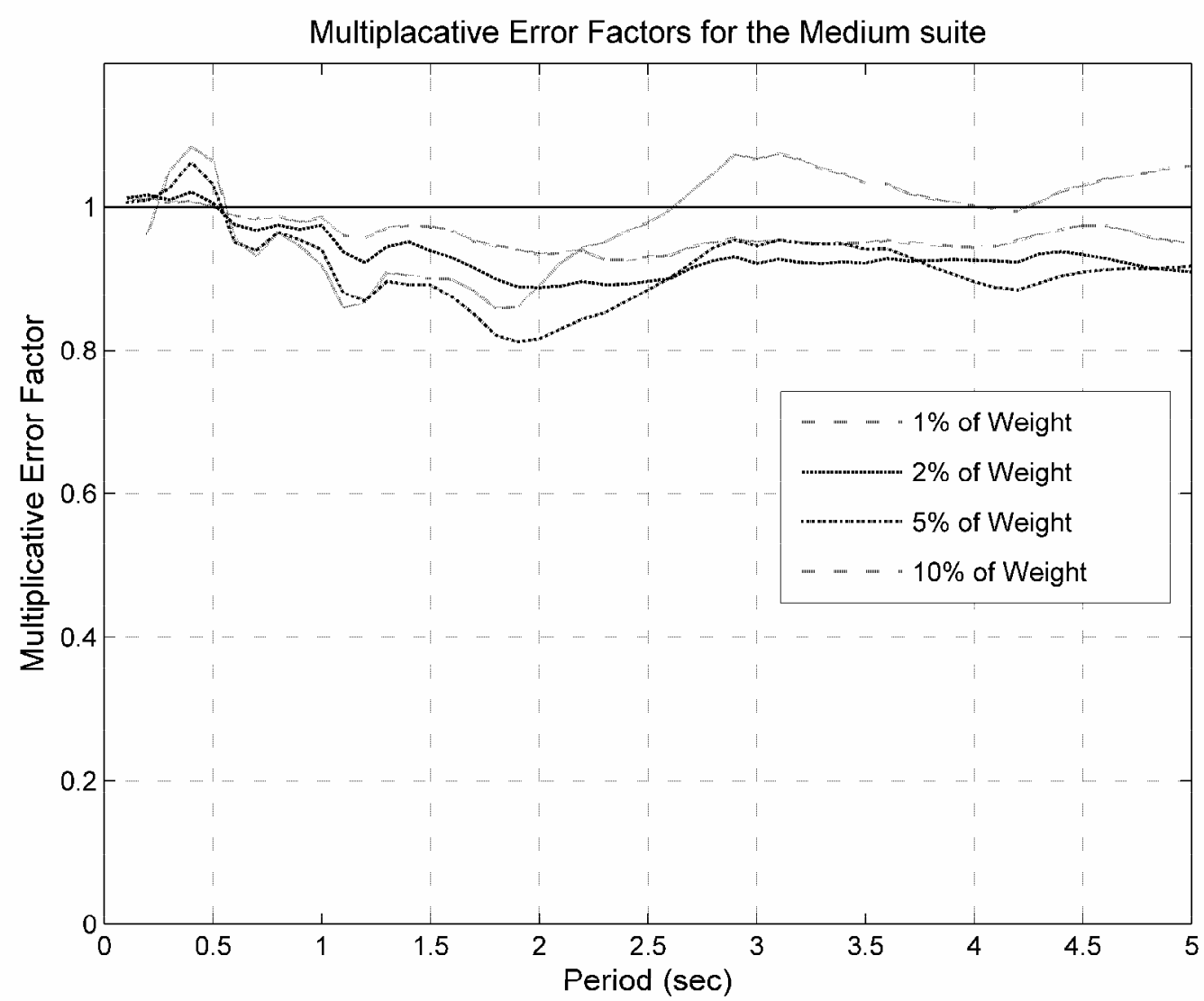

Figure 9: Multiplicative error factors for the design spectra, derived by the use of the approximate reduction factor equations, divided by the exact spectra. 


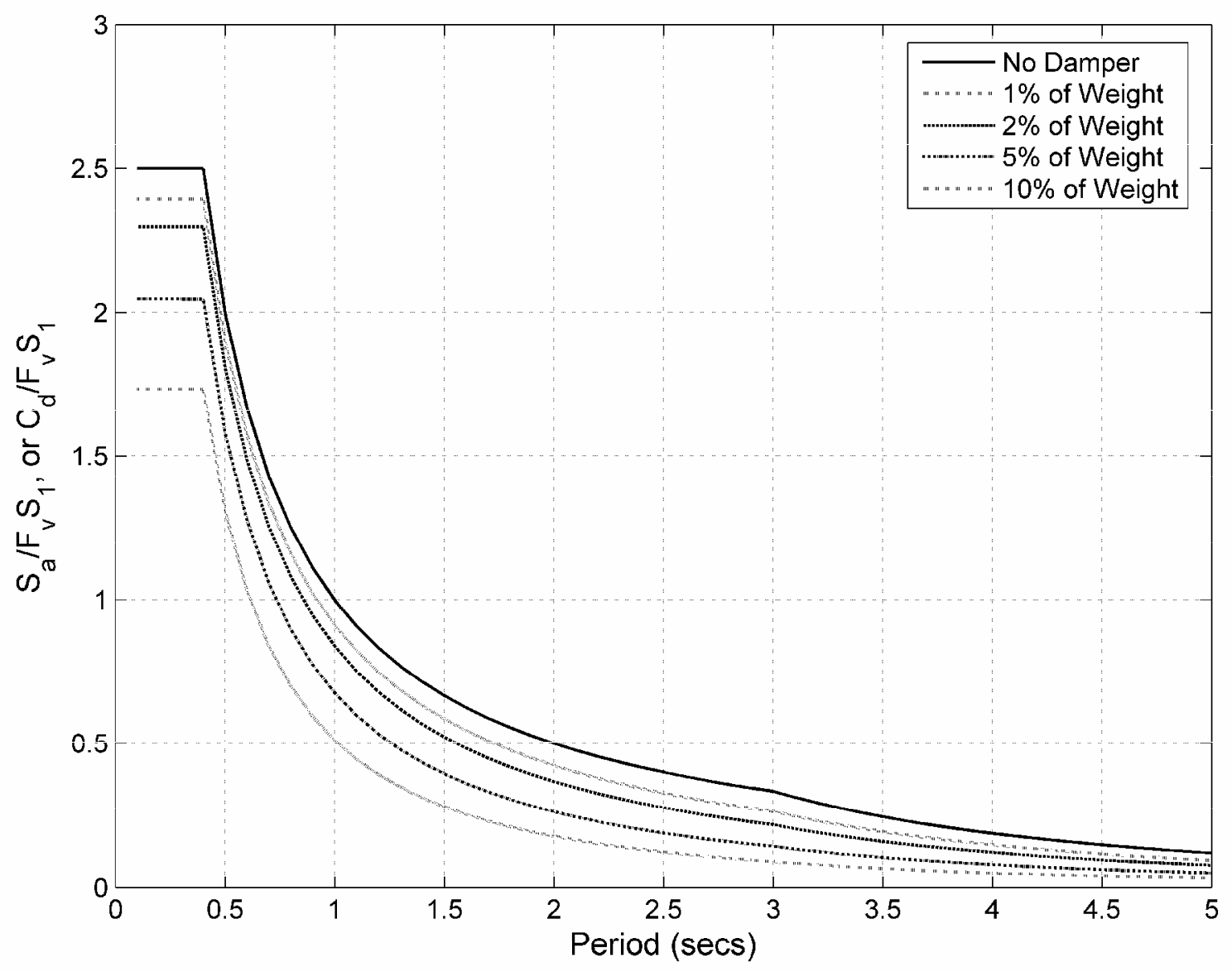

Figure 10: Normalised Design Spectra for extrusion dampers where the lateral damping force is given by $\varepsilon=$ percentage of seismic weight. 


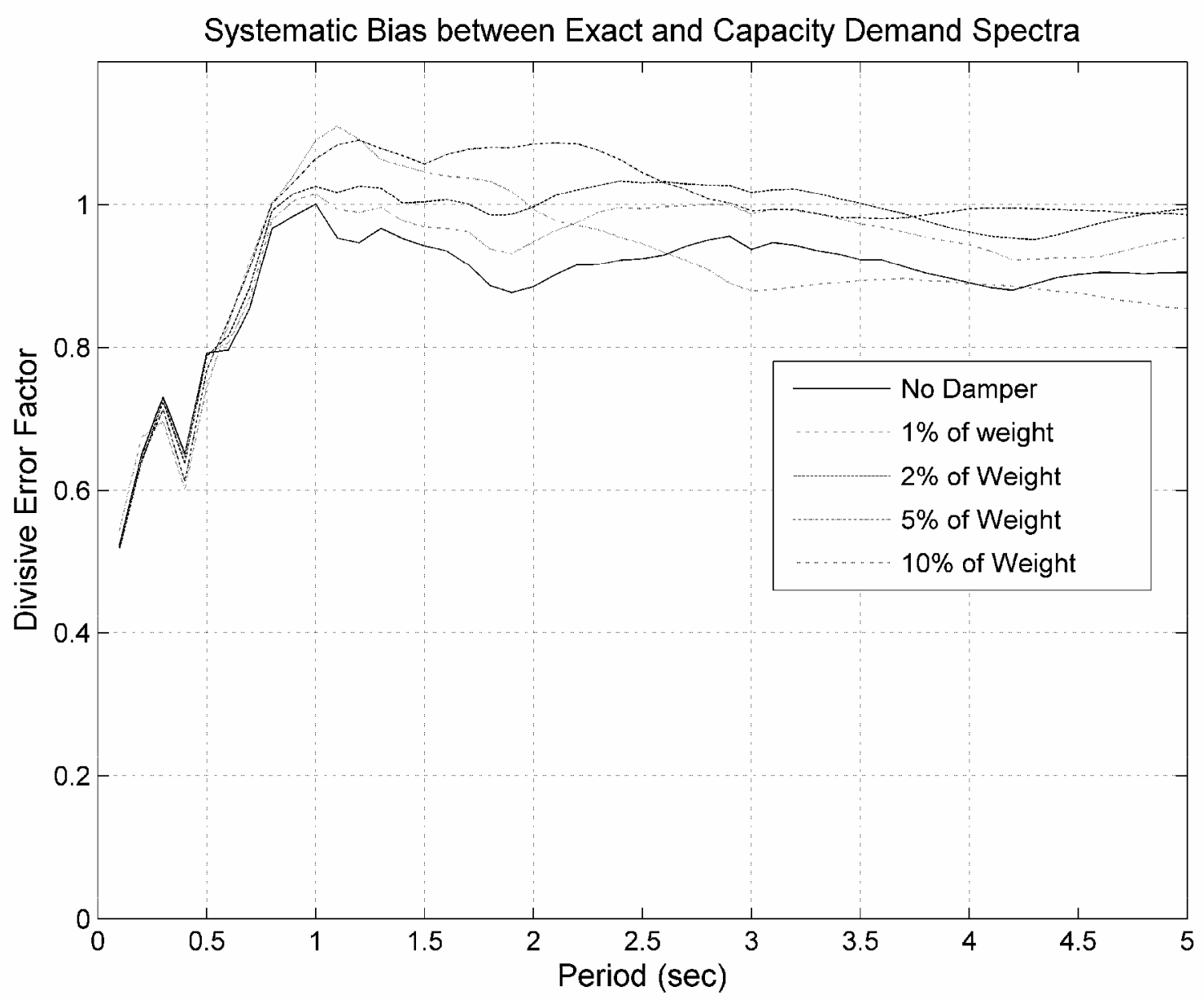

Figure 11: Systematic bias between the exact spectra and the capacity-demand spectra (shown in Figure 10) with $F_{v} S_{l}=0.68$. Note that values less than 1.0 imply conservatism. 